\title{
CARACTERIZAÇÃO HIDRÁULICA DE UM TUBO GOTEJADOR
}

\author{
José Antonio Frizzone', Angelita Teresinha Vieira ${ }^{2}$, Vital Pedro da Silva Paz \\ e Tarlei Arriel Botrel ${ }^{1}$
}

\begin{abstract}
RESUMO
Foram estudadas as características hidráulicas do tubo gotejador Rain Bird, constituído de polietileno flexível, parede com 225 microns de espessura, emissores tipo labirinto integrados à parede do tubo e espaçados $0,30 \mathrm{~m}$. O tubo gotejador foi submetido a diferentes pressões para a determinação das características hidráulicas: relação vazão-pressão dos emissores, coeficiente de variação de fabricação, resistência à ruptura, perda de carga, fator de redução de perda de carga e comprimento máximo para linhas laterais. Os emissores apresentam valor médio de coeficiente de variação de fabricação de $1,97 \%$ e regime de fluxo turbulento $(\mathrm{x}=0,4563)$. Verificou-se aumento do diâmetro interno do tubo em função do aumento da pressão, e a ruptura ocorreu a pressão de $206,0 \mathrm{kPa}$, enquanto o coeficiente de redução de perda de carga diminuiu com o aumento da pressão para um mesmo número de emissores e o comprimento máximo da linha lateral, obtido pelo método estatístico, apresentou grande dependência da pressão de entrada na tubulação, da diferença de nível e do coeficiente de variação de pressão ao longo da mesma.
\end{abstract}

Palavras-chave: irrigação, hidráulica, tubo gotejador

HYDRAULIC CHARACTERIZATION OF DRIP TAPE

\begin{abstract}
The hydraulic performance of Rain Bird drip tape was evaluated. The drip tape is made of flexible polyethylene with 225 microns wall thickness and long path emitters spaced at $0,30 \mathrm{~m}$. The drip tape was tested with different inlet pressures to determine the hydraulic characteristics: coefficient of manufacturing variation, emitter flow equation, rupture resistance, friction head loss equation, friction head loss factor and maximum lateral length. The average coefficient of manufacturing variation was $1,97 \%$ and turbulent flow was observed on the emitter (discharge exponent 0,4563 ). The internal diameter of drip tape increased with the increase in pressure and the rupture resistance failed at 206,0kPa. The value of friction head loss factor decreased as the pressure increased to the same number of emitters. The maximum lateral length obtained by the statistical approach was found to be highly dependent on the inlet pressure head, the slope of the lateral and the coefficient of variation of pressure head along the lateral line.
\end{abstract}

Key words: irrigation, hydraulic, drip tape

\footnotetext{
${ }^{1}$ Prof. Associado do Depto. Eng. Rural, ESALQ/USP, Av. Pádua Dias 11. CEP 13418-900, Piracicaba, SP. Fone (019)429-4217 E-mail:frizzone@carpa.ciagri.usp.br

${ }^{2}$ M.Sc. Irigação e Drenagem, Depto. Eng. Rural, ESALQ/USP

${ }^{3}$ Doutor em Irrigação e Drenagem, ESALQ-FAPESP, Av. Pádua Dias 11. CEP 13418-900, Piracicaba, SP. E-mail: vpspaz@carpa.ciagri.usp.br, Fone (019)429-4217
} 


\section{INTRODUÇÃO}

Para se reduzir o investimento de capital na aquisição do sistema de irrigação por gotejamento, estão sendo comercializados tubos gotejadores confeccionados com polietileno flexível e parede de pequena espessura. Os emissores de água, tipo labirinto, são integrados à parede do tubo, em espaçamentos regulares. Em decorrência do tipo de material utilizado, do diâmetro do tubo, da espessura da parede e da geometria do emissor, torna-se importante analisar as características hidráulicas do tubo gotejador, visando fornecer elementos para o projeto e para a operação do sistema de irrigação.

Solomon (1979) relata sobre a importância de se considerar a variação da vazão dos gotejadores em função da variação na fabricação. Vermeiren \& Jobling (1980) e Botrel (1984) destacam que pequenas variações na geometria dos orifícios de saída da água provocam sensíveis diferenças na vazão assumindo, em alguns casos, maior importância que as variações de vazão decorrentes das diferenças de pressão ao longo da linha lateral.

Admitindo-se que as variações de vazão ocasionadas pelo processo construtivo têm distribuição normal, é possível utilizarse conceitos da estatística clássica para análises quantitativas. Solomon (1979) estudando as variações da fabricação de vários emissores tipo orifício, classificou e atribuiu uma interpretação física ao coeficiente de variação de fabricação (CVf) relacionando o desvio-padrão da vazão dos emissores com a vazão média da amostra submetida a testes com pressão constante. Segundo o autor, o CVf é um importante parâmetro que influencia a uniformidade de emissão de água e a eficiência de aplicação de água pelo sistema.

Testezlaf \& Campioni (1993) encontraram CVf médio de $2,8 \%$ para o tubo gotejador Queen Gil, com emissores tipo labirinto, espaçados $0,30 \mathrm{~m}$, indicando uma boa qualidade de fabricação e, para o mesmo tubo gotejador, Schmidt (1995) encontrou CVf médio de 3,11\%.

A relação vazão-pressão, a perda de carga ao longo da linha lateral e no emissor e o percurso da água no mesmo, constituem as características hidráulicas que influenciam diretamente o desempenho do sistema. Essas características dependem do tipo de emissor, do material, do processo de fabricação e da forma de conexão do emissor na linha. Segundo Keller \& Karmeli (1975) a vazão de um emissor é uma função do tipo potência da

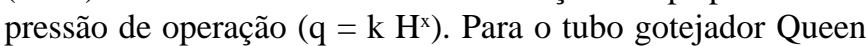
Gil, Schmidt (1995) encontrou $\mathrm{x}=0,6616$ e caracterizou o regime de escoamento como turbulento. Testezlaf \& Campioni (1993) avaliando o mesmo tubo gotejador, encontraram o valor 0,625 .

Várias equações permitem calcular a perda de carga em tubulações de polietileno de pequenos diâmetros e, dentre elas, consagrou-se a equação de Darcy-Weissbach, que pode ser utilizada em regimes laminar e turbulento, com resultados satisfatórios. O fator de atrito (f) dessa equação depende do número de Reynolds e da rugosidade relativa do tubo. Assumindo-se que os tubos de polietileno são hidraulicamente lisos, a rugosidade relativa pode ser desconsiderada.

Christiansen (1942) trabalhando com irrigação por aspersão, propôs um método para simplificar o cálculo da perda de carga em tubos com múltiplas saídas, com estas em espaçamentos equiidistantes; ele determinou um fator $(\mathrm{F})$ que, multiplicado pela perda de carga no tubo sem saídas laterais, permite determinar a perda de carga na linha lateral de irrigação. Oliveira (1978), Soares (1981), Faria (1981) e Botrel (1984) estudando a hidráulica de linhas laterais para irrigação localizada, observaram que o valor de $\mathrm{F}$ aumentou para um mesmo número de emissores, quando se diminuiu o espaçamento entre os mesmos. Andrade (1990) verificou que, para um mesmo espaçamento entre saídas laterais, o valor de $\mathrm{F}$ diminuiu quando se aumentou o número de emissores e cresceu quando a pressão foi também aumentada, a partir de um número de emissores.

Vários métodos de dimensionamento de linhas laterais são conhecidos, considerando-se apenas as variações de pressão ao longo da linha lateral, por efeito de perdas de carga ou diferença de nível. Anyoji \& Wu (1987) desenvolveram uma técnica para o cálculo do comprimento máximo de linhas laterais, usando aproximação estatística, em que consideraram as variações de vazão por efeitos hidráulicos (perda de carga e diferença de nível) e construtivos dos emissores. Expressaram as variações de vazão do emissor como coeficiente de variação de vazão $(\mathrm{CVq})$ em função do coeficiente de variação de fabricação (CVf) e do coeficiente de variação de pressão ao longo da linha lateral (CVh). Considerando-se k e H da equação de fluxo do emissor como variáveis aleatórias independentes, uma equação de vazão média foi derivada. Estabelecendo-se o CVq como critério de projeto, o CVh para a linha lateral pode ser calculado e o seu comprimento determinado.

O objetivo deste trabalho foi determinar as características hidráulicas do tubo gotejador Rain-Tape TPC, analisando-se a uniformidade de vazão, a equação característica do emissor, a variação do diâmetro interno, a resistência do material à pressão, a perda de carga e o comprimento máximo de linhas laterais.

\section{MATERIAL E MÉTODOS}

Este trabalho foi realizado no Laboratório de Irrigação do Departamento de Engenharia Rural-ESALQ/USP, utilizandose o tubo gotejador Rain-Tape TPC, fabricado pela Rain Bird, de polietileno linear de baixa densidade, parede com 225 microns de espessura e emissores autocompensantes, tipo labirinto, espaçados de $0,30 \mathrm{~m}$

Os emissores foram submetidos a ensaios para análise da uniformidade de vazão, determinando-se o coeficiente de variação de fabricação (CVf) e as pressões de ensaio variaram de $19,6 \mathrm{kPa}$ a $98,1 \mathrm{kPa}$, sendo determinadas com manômetro de coluna líquida conectado ao segmento da tubulação em teste. Mediu-se a vazão correspondente a cada pressão de operação para se obter a relação entre essas variáveis, enquanto para cálculo da vazão média e do desvio padrão em cada pressão de ensaio, foram consideradas duas repetições de 52 emissores, utilizando-se 4 segmentos de tubo com 13 emissores cada um; já o CVf foi obtido pela média dos valores de cada pressão.

O diâmetro do tubo gotejador foi medido com o auxílio de um paquímetro digital com resolução de $10^{-5} \mathrm{~m}$, a cada pressão de operação, em três locais distintos (início, meio e fim) do segmento de tubulação. A média desses valores foi relacionada à pressão correspondente por análise de regressão, ajustando-se a uma equação potencial. 
Determinou-se a resistência do tubo gotejador a pressão, submetendo-o a pressões internas gradualmente crescentes; utilizaram-se 10 segmentos, com $5 \mathrm{~m}$ de comprimento cada um e manômetro digital, com resolução de $9,8 \mathrm{kPa}$, instalado na linha-teste, possibilitando a leitura da pressão no momento da ruptura. Para cada incremento de pressão considerou-se um período de tempo de até 10 minutos, para possível início do processo de ruptura.

Para a determinação da perda de carga ao longo do tubo, utilizou-se um segmento de 50m de comprimento, em nível, com todos os emissores vedados; foram conectadas tomadas de pressão, no início e no final desse segmento, e a perda de carga obtida pela diferença de pressão entre os pontos, medida em manômetros diferenciais, enquanto a tubulação de testes foi submetida a quatro pressões de entrada $(29,2 \mathrm{kPa}, 58,9 \mathrm{kPa}$, $78,5 \mathrm{kPa}$ e $88,3 \mathrm{kPa}$ ) ajustadas por uma válvula reguladora de pressão, seguida de um medidor de vazão e um manômetro de coluna de líquido. No final da tubulação, foi instalado um registro para ajuste da vazão e 40 pares de valores de vazão e perda de carga foram obtidos em cada pressão e, por análise de regressão, estabeleceu-se a equação de perda de carga.

$\mathrm{O}$ fator de redução de perda de carga $(\mathrm{F})$ foi determinado para o tubo gotejador pela relação entre a perda de carga, considerando-se o tubo com múltiplas saídas laterais e a perda de carga no tubo sem saídas laterais. A perda de carga no tubo com múltiplas saídas foi calculada utilizando-se o método trecho-a-trecho, conforme apresenta Botrel (1984) simulandose uma linha lateral com $\mathrm{N}$ emissores $(\mathrm{N}=2,3, \ldots, 700)$ submetida a diferentes pressões de entrada $(19,6 \mathrm{kPa}$, $29,4 \mathrm{kPa}, \ldots, 98,1 \mathrm{kPa})$.

O cálculo do comprimento máximo da linha lateral foi feito pelo método estatístico, seguindo-se o procedimento desenvolvido por Anyoji \& Wu (1987) a saber:

a) para um coeficiente de variação total da vazão $(\mathrm{CVq})$ e se conhecendo os valores de x e CVf, calcula-se o coeficiente de variação de vazão decorrente da variação de pressão (CVh), considerada critério de projeto:

$$
\mathrm{CVq}=\frac{\sqrt{\mathrm{CVf}^{2}+\mathrm{x}^{2} \mathrm{CVh}_{\mathrm{p}}^{2}}}{1+\mathrm{CVh}_{\mathrm{p}}^{2}\left(0,5 \mathrm{x}^{2}-0,5 \mathrm{x}\right)}
$$

em que $\mathrm{CVh}_{\mathrm{p}}$ é o coeficiente de variação de pressão permitido ao longo da linha lateral;

b) conhecendo-se os valores do expoente de fluxo do emissor (x) do $\mathrm{CVh}_{\mathrm{p}}$ e da vazão média do emissor $\left(\mathrm{q}_{\mathrm{m}}\right)$ calcula-se a pressão média $\left(\mathrm{H}_{\mathrm{m}}\right)$ de operação, utilizando-se a expressão:

$$
H_{m}=\left\{\frac{q_{m}}{k\left[1+C V h_{p}^{2}\left(0,5 x^{2}-0,5 x\right)\right]}\right\}^{1 / x}
$$

c) atribui-se valor ao comprimento (L) da lateral e calculase a perda de carga (Hf):

$$
\mathrm{Hf}=\frac{1}{\mathrm{~m}+1} \mathrm{a} \mathrm{Q}^{\mathrm{m}} \mathrm{L}
$$

d) com os valores de Hf e a diferença de nível (DZ) calculase a variância da pressão [V(h)] ao longo da lateral:

$V(h)=\frac{(m+1)^{2}}{(2 m+3)(m+2)^{2}} H f^{2}+\frac{1}{12} \Delta Z^{2}+\frac{m+1}{(m+2)(m+3)} H f \Delta Z$ sendo $\Delta \mathrm{Z}$ negativo para tubulação em declive e positivo para aclive:

e) com os valores de $\mathrm{V}(\mathrm{h})$ e $\mathrm{H}_{\mathrm{m}}$, calcula-se o coeficiente de variação da pressão na lateral de comprimento L:

$$
\mathrm{CVh}=\frac{\sqrt{\mathrm{V}(\mathrm{h})}}{\mathrm{H}_{\mathrm{m}}}
$$

f) comparam-se os valores de $\mathrm{CVh}_{\mathrm{p}}$ e $\mathrm{CVh}$, em que: se iguais, o comprimento máximo está determinado e, se diferentes, atribui-se novo valor a $L$ e se repetem os cálculos a partir de (c).

No presente trabalho, o comprimento máximo da lateral foi calculado para CVh igual a 3,7\% e $7,8 \%$, para pressões de entrada variando de $19,6 \mathrm{kPa}$ a $98,1 \mathrm{kPa}$.

\section{RESULTADOS E DISCUSSÃO}

\section{Coeficiente de variação e relação vazão-pressão}

$\mathrm{O}$ efeito dos fatores construtivos dos emissores do tubo gotejador Rain-Tape TPC, proporcionou coeficientes de variação de fabricação (CVf) de 1,74\% a 2,4\%, com valor médio de $1,97 \%$ (Tabela 1) inferior ao informado pelo fabricante $(4 \%)$. Segundo a classificação proposta pelo USDA (1979) e Solomon (1979) a uniformidade de fabricação é excelente e, de acordo com a ABNT (1986) é boa. Por análise de regressão obteve-se a relação vazão-pressão para o emissor, cujo modelo está representado na Figura 1 . O valor $\mathrm{x}=0,4563$ indica que o regime de escoamento pode ser considerado turbulento; já no intervalo de pressões de $49,0 \mathrm{kPa}$ a $98,1 \mathrm{kPa}$, cuja operação é indicada pelo fabricante, as vazões estimadas pela equação ajustada foram $0,5 \%$ a $6,4 \%$ superiores aos valores de catálogo.

Tabela 1. Vazão média, desvio-padrão e coeficiente de variação de fabricação para emissores do tubo gotejador Rain-Tape, submetido a diferentes pressões

\begin{tabular}{cccc}
\hline Pressão $(\mathrm{kPa})$ & Vazão média (1/h) & Desvio-padrão & CVf (\%), \\
\hline 19,6 & 0,633 & 0,015 & 2,40 \\
29,4 & 0,769 & 0,017 & 2,19 \\
39,2 & 0,880 & 0,017 & 1,92 \\
49,0 & 0,974 & 0,018 & 1,88 \\
58,9 & 1,056 & 0,019 & 1,81 \\
68,6 & 1,132 & 0,022 & 1,96 \\
78,5 & 1,199 & 0,023 & 1,88 \\
88,3 & 1,265 & 0,022 & 1,74 \\
98,1 & 1,321 & 0,025 & 1,92 \\
\hline Média & & & 1,97 \\
\hline
\end{tabular}

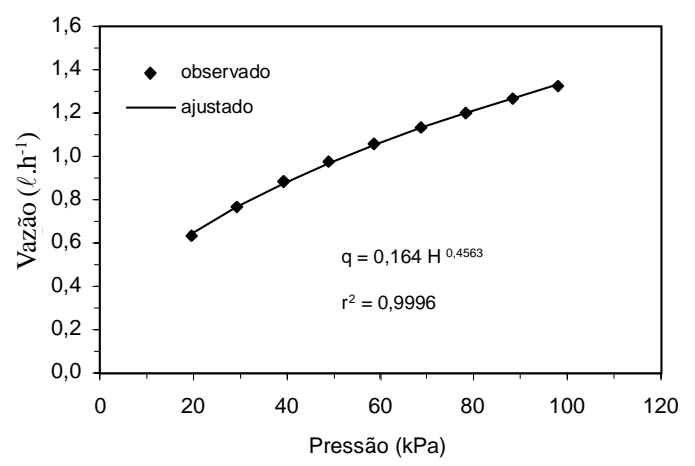

Figura 1. Relação vazão-pressão para o emissor do tubo gotejador Rain-Tape 


\section{Diâmetro interno}

A análise de variância indicou haver diferença significativa a 5\% de probabilidade, entre as médias dos diâmetros internos do tubo nas diferentes pressões, apesar da diferença percentual entre o menor e maior diâmetro não ter sido superior a $1 \%$. O teste de Tukey indicou quatro grupos de valores que podem ser considerados homogêneos (Tabela 2) a nível de 5\% de probabilidade. A variação do diâmetro com a pressão é representada pelo modelo potencial mostrado na Figura 2. Andrade (1990) determinando as características hidráulicas de tubos flexíveis de polietileno perfurados a laser, também verificou que o aumento da pressão resultou em crescimento do diâmetro interno e estabeleceu uma relação potencial entre diâmetro e pressão.

Tabela 2. Valores médios do diâmetro interno do tubo gotejador a diferentes pressões

\begin{tabular}{cccc}
\hline $\begin{array}{c}\text { Pressão } \\
(\mathrm{kPa})\end{array}$ & $\mathrm{N}$ & $\begin{array}{c}\text { Diâmetro médio } \\
(\mathrm{m})\end{array}$ & $\begin{array}{c}\text { Grupos } \\
\text { homogêneos* }\end{array}$ \\
\hline 19,6 & 12 & $16,66 \times 10^{-3}$ & $\mathrm{a}$ \\
29,4 & 12 & $16,69 \times 10^{-3}$ & $\mathrm{a} \mathrm{b}$ \\
39,2 & 12 & $16,71 \times 10^{-3}$ & $\mathrm{a} \mathrm{b} \mathrm{c}$ \\
49,0 & 12 & $16,71 \times 10^{-3}$ & $\mathrm{a} \mathrm{b} \mathrm{c}$ \\
58,9 & 12 & $16,74 \times 10^{-3}$ & $\mathrm{~b} \mathrm{c} \mathrm{d}$ \\
68,6 & 12 & $16,77 \times 10^{-3}$ & $\mathrm{~b} \mathrm{c} \mathrm{d}$ \\
78,5 & 12 & $16,78 \times 10^{-3}$ & $\mathrm{c} \mathrm{d}$ \\
88,3 & 12 & $16,79 \times 10^{-3}$ & $\mathrm{~d}$ \\
98,1 & 12 & $16,81 \times 10^{-3}$ & $\mathrm{~d}$ \\
\hline
\end{tabular}

*Médias seguidas de mesma letra não diferem, a $5 \%$ de probabilidade.

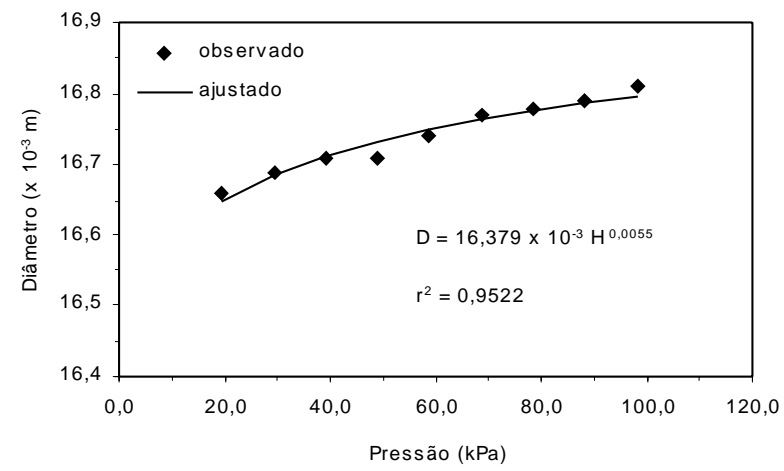

Figura 2. Diâmetro interno do tubo gotejador em função da pressão de operação

\section{Resistência do tubo gotejador}

Nos testes de resistência do tubo gotejador, a pressão de ruptura manteve-se praticamente constante, em torno de $206,0 \mathrm{kPa}$, enquanto a ruptura ocorreu em locais distintos, ao longo da tubulação. A partir de $166,8 \mathrm{kPa}$ o tubo começou a deformar-se ao longo dos $5 \mathrm{~m}$, observando-se a formação de uma "vesícula" com parede pouco espessa, que se rompeu em seguida. O processo de ruptura sempre ocorreu na parede do tubo gotejador e não nos locais onde estão integrados os emissores, permanecendo os mesmos gotejando durante o período de deformação do tubo. Testezlaf \& Campioni (1993) encontraram pressão média de ruptura de 382,6kPa para o tubo gotejador Queen Gil e verificaram, também, que o número de emissores na linha não afetou significativamente a pressão de ruptura.

\section{Perda de carga}

Por existir variações do diâmetro interno da tubulação em função da pressão, ajustaram-se equações de perda de carga, em função da vazão, para quatro pressões distintas: $39,2 \mathrm{kPa}$, $58,9 \mathrm{kPa}, 78,5 \mathrm{kPa}$ e $88,3 \mathrm{kPa}$, que resultaram nas seguintes expressões:

$$
\begin{array}{ll}
\mathrm{J}_{39,2}=259,708 \times 10^{3} \mathrm{Q}^{1,7716} & \mathrm{r}^{2}=0,9998 \\
\mathrm{~J}_{58,9}=194,370 \times 10^{3} \mathrm{Q}^{1,7431} & \mathrm{r}^{2}=0,9995 \\
\mathrm{~J}_{78,5}=183,394 \times 10^{3} \mathrm{Q}^{1,7398} & \mathrm{r}^{2}=0,9995 \\
\mathrm{~J}_{88,3}=188,685 \times 10^{3} \mathrm{Q}^{1,7447} & \mathrm{r}^{2}=0,9995
\end{array}
$$

em que:

$\mathrm{J}=$ perda de carga unitária, $\mathrm{m} \cdot \mathrm{m}^{-1}$

$\mathrm{Q}=$ vazão, $\mathrm{m}^{3} \cdot \mathrm{s}^{-1}$.

Para se verificar a possibilidade da perda de carga ser representada por uma única expressão, ajustou-se, independente da pressão, a seguinte equação:

$$
J=190,977 \times 10^{3} Q^{1,7423} \quad r^{2}=0,9987
$$

As equações (6), (7), (8), (9) e (10) foram submetidas ao teste "Fz", segundo Zar (1984) para comparação de regressões, pelo qual se verificou diferença significativa, a nível de 5\% de probabilidade, entre as mesmas. Em razão do efeito da pressão no diâmetro da tubulação e, consequentemente, na perda de carga, ajustou-se uma equação de perda de carga em função da vazão e do diâmetro, além de outra, em função da vazão e da pressão (equações 11 e 12):

$$
\begin{gathered}
J=7,1509.10^{-4} Q^{1,7359} D^{-4,7332} \quad r^{2}=0,9994 \\
J=363,179 \times 10^{-7} Q^{1,7642} H^{-0,1079} \quad r^{2}=0,9998
\end{gathered}
$$

sendo:

J - perda de carga unitária no tubo gotejador, $\mathrm{m} \cdot \mathrm{m}^{-1}$

$\mathrm{Q}$ - vazão, $\mathrm{m}^{3} \cdot \mathrm{s}^{-1}$

$\mathrm{H}$ - pressão de operação, $\mathrm{kPa}$

D - diâmetro do tubo gotejador, $m$

A análise de variância para perda de carga medida e estimada pelas Equações (11) e (12) e pela equação de Darcy-Weissbach, com $\mathrm{f}$ calculado pela fórmula de Blasius e diâmetro ajustado em função da pressão, indicou não haver diferença significativa entre elas pelo teste $\mathrm{F}$, a $5 \%$ de probabilidade; pode-se, portanto, indicar qualquer uma dessas equações para estimativa da perda de carga no tubo gotejador Rain-Tape, da Rain Bird.

Para o cálculo de $\mathrm{F}$ utilizou-se a equação ajustada para a relação vazão-pressão e a Equação (12) para a perda de carga. Para uma pressão de operação constante, os valores de F diminuíram com o aumento do número de emissores (Tabela 3); entretanto, para um mesmo número de emissores, os valores de F diminuíram com o aumento da pressão, diminuição esta que pode estar associada ao crescimento do diâmetro interno da tubulação, decorrente da elevação da pressão, embora Andrade (1990) tenha observado aumento dos valores de F com o progresso da pressão. 
Tabela 3. Valores de F para o tubo gotejador Rain-Tape, com emissores espaçados de 0,30m, em função do número de saídas (N) e da pressão de operação

\begin{tabular}{|c|c|c|c|c|c|c|c|c|c|}
\hline \multirow{2}{*}{$\begin{array}{l}\text { Saídas } \\
(\mathrm{N})\end{array}$} & \multicolumn{9}{|c|}{ Pressão de operação $(\mathrm{kPa})$} \\
\hline & 19,6 & 29,4 & 39,2 & 49,0 & 58,9 & 68,6 & 78,5 & 88,3 & 98,1 \\
\hline 1 & 1 & 1 & 1 & 1 & 1 & 1 & 1 & 1 & 1 \\
\hline 2 & 0,647 & 0,626 & 0,611 & 0,600 & 0,591 & 0,584 & 0,578 & 0,572 & 0,568 \\
\hline 3 & 0,544 & 0,523 & 0,509 & 0,498 & 0,489 & 0,485 & 0,475 & 0,470 & 0,465 \\
\hline 4 & 0,496 & 0,476 & 0,462 & 0,451 & 0,443 & 0,436 & 0,423 & 0,425 & 0,420 \\
\hline 5 & 0,468 & 0,448 & 0,435 & 0,425 & 0,417 & 0,410 & 0,404 & 0,399 & 0,395 \\
\hline 6 & 0,449 & 0,430 & 0,417 & 0,408 & 0,400 & 0,393 & 0,388 & 0,383 & 0,379 \\
\hline 7 & 0,436 & 0,418 & 0,405 & 0,396 & 0,388 & 0,382 & 0,376 & 0,372 & 0,367 \\
\hline 8 & 0,427 & 0,408 & 0,396 & 0,387 & 0,379 & 0,373 & 0,368 & 0,363 & 0,359 \\
\hline 9 & 0,419 & 0,401 & 0,389 & 0,380 & 0,373 & 0,366 & 0,361 & 0,357 & 0,353 \\
\hline 10 & 0,413 & 0,396 & 0,384 & 0,375 & 0,363 & 0,361 & 0,356 & 0,352 & 0,348 \\
\hline 11 & 0,408 & 0,391 & 0,379 & 0,371 & 0,359 & 0,357 & 0,352 & 0,347 & 0,353 \\
\hline 12 & 0,404 & 0,387 & 0,375 & 0,367 & 0,356 & 0,353 & 0,348 & 0,344 & 0,340 \\
\hline 13 & 0,401 & 0,384 & 0,372 & 0,363 & 0,354 & 0,351 & 0,346 & 0,341 & 0,337 \\
\hline 14 & 0,398 & 0,381 & 0,370 & 0,361 & 0,352 & 0,348 & 0,343 & 0,339 & 0,335 \\
\hline 15 & 0,396 & 0,379 & 0,367 & 0,359 & 0,350 & 0,346 & 0,341 & 0,337 & 0,333 \\
\hline 16 & 0,394 & 0,377 & 0,365 & 0,357 & 0,348 & 0,344 & 0,339 & 0,335 & 0,331 \\
\hline 17 & 0,392 & 0,375 & 0,364 & 0,355 & 0,346 & 0,342 & 0,337 & 0,333 & 0,329 \\
\hline 18 & 0,390 & 0,373 & 0,362 & 0,353 & 0,345 & 0,341 & 0,336 & 0,332 & 0,328 \\
\hline 19 & 0,389 & 0,372 & 0,361 & 0,352 & 0,344 & 0,339 & 0,335 & 0,330 & 0,327 \\
\hline 20 & 0,387 & 0,371 & 0,359 & 0,351 & 0,343 & 0,338 & 0,333 & 0,329 & 0,326 \\
\hline 25 & 0,382 & 0,366 & 0,355 & 0,346 & 0,339 & 0,334 & 0,329 & 0,325 & 0,321 \\
\hline 30 & 0,379 & 0,362 & 0,351 & 0,343 & 0,336 & 0,331 & 0,326 & 0,322 & 0,318 \\
\hline 35 & 0,376 & 0,360 & 0,349 & 0,341 & 0,334 & 0,329 & 0,324 & 0,320 & 0,316 \\
\hline 40 & 0,374 & 0,358 & 0,347 & 0,339 & 0,333 & 0,327 & 0,322 & 0,318 & 0,315 \\
\hline 50 & 0,372 & 0,356 & 0,345 & 0,337 & 0,330 & 0,325 & 0,320 & 0,316 & 0,313 \\
\hline 60 & 0,370 & 0,354 & 0,343 & 0,335 & 0,329 & 0,323 & 0,319 & 0,315 & 0,311 \\
\hline 70 & 0,369 & 0,353 & 0,342 & 0,334 & 0,328 & 0,322 & 0,318 & 0,314 & 0,310 \\
\hline 80 & 0,368 & 0,352 & 0,341 & 0,333 & 0,327 & 0,321 & 0,317 & 0,313 & 0,309 \\
\hline 90 & 0,367 & 0,352 & 0,341 & 0,333 & 0,336 & 0,321 & 0,316 & 0,312 & 0,309 \\
\hline 100 & 0,367 & 0,351 & 0,340 & 0,332 & 0,326 & 0,320 & 0,316 & 0,312 & 0,308 \\
\hline 120 & 0,366 & 0,350 & 0,339 & 0,331 & 0,335 & 0,320 & 0,315 & 0,311 & 0,307 \\
\hline 140 & 0,365 & 0,349 & 0,339 & 0,331 & 0,324 & 0,319 & 0,314 & 0,310 & 0,307 \\
\hline 160 & 0,364 & 0,349 & 0,338 & 0,330 & 0,324 & 0,318 & 0,314 & 0,310 & 0,306 \\
\hline 180 & 0,364 & 0,348 & 0,337 & 0,329 & 0,323 & 0,318 & 0,313 & 0,309 & 0,306 \\
\hline 200 & 0,363 & 0,347 & 0,337 & 0,329 & 0,323 & 0,317 & 0,313 & 0,309 & 0,305 \\
\hline 300 & 0,359 & 0,344 & 0,334 & 0,326 & 0,320 & 0,315 & 0,310 & 0,307 & 0,303 \\
\hline 400 & 0,353 & 0,339 & 0,329 & 0,322 & 0,316 & 0,311 & 0,307 & 0,303 & 0,300 \\
\hline 500 & 0,346 & 0,332 & 0,323 & 0,316 & 0,311 & 0,306 & 0,302 & 0,299 & 0,296 \\
\hline 600 & 0,336 & 0,324 & 0,316 & 0,309 & 0,304 & 0,300 & 0,296 & 0,293 & 0,290 \\
\hline 700 & 0,325 & 0,314 & 0,307 & 0,301 & 0,297 & 0,293 & 0,289 & 0,286 & 0,284 \\
\hline
\end{tabular}

Comprimento máximo de linhas laterais

Para o dimensionamento de linhas laterais pelo método estatístico consideraram-se valores de CVh de 3,7\% e 7,8\% pois, segundo Sammis \& Wu (1985) correspondem a variações de vazão $\left.\left[\mathrm{q}_{\text {var }}=\left(\mathrm{q}_{\max }-\mathrm{q}_{\min }\right) / \mathrm{q}_{\max }\right)\right]$ da ordem de $10 \%$ e $20 \%$, respectivamente, para emissores de fluxo turbulento. Os comprimentos máximos de linhas laterais para o tubo gotejador
Rain-Tape, obtidos para pressões de entrada variando de $19,6 \mathrm{kPa}$ a $98,1 \mathrm{kPa}$ e tubulação em nível, em aclive de $1 \%$ e $2 \%$ e declive de $1 \%$ e $2 \%$, estão apresentados na Tabela 4.

Observa-se que para tubulação em nível e aclive, o comprimento máximo sempre cresceu com o aumento da pressão de entrada até $98,1 \mathrm{kPa}$, porém com taxas de acréscimos decrescentes, e a linha em nível proporcionou maiores 
Tabela 4. Comprimento máximo de linhas laterais (m) para o tubo gotejador Rain-Tape

\begin{tabular}{|c|c|c|c|c|c|c|c|c|c|c|}
\hline \multirow{2}{*}{$\begin{array}{l}\text { CVh } \\
(\%)\end{array}$} & \multirow{2}{*}{$\begin{array}{l}\text { So* } \\
(\%)\end{array}$} & \multicolumn{9}{|c|}{ Pressão de entrada $(\mathrm{kPa})$} \\
\hline & & 19,6 & 29,4 & 39,2 & 49,0 & 58,9 & 68,6 & 78,5 & 88,3 & 98,1 \\
\hline 3,7 & +2 & 13,2 & 19,5 & 25,8 & 31,5 & 37,2 & 42,6 & 47,4 & 51,9 & 56,1 \\
\hline 7,8 & +2 & 27,0 & 39,3 & 50,7 & 60,6 & 69,6 & 77,1 & 83,7 & 89,7 & 94,8 \\
\hline 3,7 & +1 & 25,5 & 36,9 & 46,5 & 54,6 & 61,2 & 66,9 & 71,7 & 75,9 & 79,5 \\
\hline 7,8 & +1 & 50,1 & 67,8 & 80,7 & 90,6 & 98,4 & 104,7 & 109,8 & 114,0 & 117,9 \\
\hline 3,7 & 0 & 92,1 & 96,3 & 99,3 & 101,7 & 103,8 & 105,6 & 107,1 & 108,6 & 109,8 \\
\hline 7,8 & 0 & 120,0 & 125,4 & 129,3 & 132,6 & 135,3 & 137,7 & 139,5 & 141,3 & 143,1 \\
\hline 3,7 & -1 & 27,0 & 43,5 & 71,1 & 144,3 & 143,1 & 141,6 & 140,4 & 139,5 & 138,6 \\
\hline 7,8 & -1 & 63,0 & 177,6 & 174,9 & 172,2 & 170,4 & 169,2 & 168,3 & 167,7 & 167,4 \\
\hline 3,7 & -2 & 13,2 & 20,1 & 27,0 & 34,2 & 42,3 & 51,9 & 63,9 & 90,6 & 156,9 \\
\hline 7,8 & -2 & 27,9 & 42,9 & 60,6 & 87,6 & 193,2 & 192,9 & 191,4 & 189,9 & 188,4 \\
\hline
\end{tabular}

*Desnível (positivo para tubulação em aclive e negativo para declive)

comprimentos; já para condições de declive, as relações entre comprimento máximo e pressão de entrada não apresentam a mesma tendência, pois existem três tipos de perfis de pressão, ao longo da tubulação, que dependem da relação entre perda de carga e diferença de nível, conforme mostram Gillespie et al. (1979). Verifica-se que, para uma pressão de entrada e dependendo do grau de declive, ocorre aumento considerável do comprimento máximo da tubulação e, nas pressões subsequientes, o comprimento tende a diminuir, porém com pequenas taxas de redução, o que pode ser verificado, por exemplo, para o declive de $1 \%$ com $\mathrm{CVh}=3,7 \%$, em que o comprimento máximo aumentou de $71,1 \mathrm{~m}$ para $144,3 \mathrm{~m}$, quando a pressão aumentou de $39,2 \mathrm{kPa}$ para $49,0 \mathrm{kPa}$, e nas pressões superiores ocorreu pequena redução. Este aumento brusco ocorre em razão da mudança no tipo de perfil de pressão, influenciado pela relação entre o declive e a perda de carga.

\section{CONCLUSÕES}

1. Os emissores do tubo gotejador apresentam uniformidade de vazão excelente e regime de escoamento turbulento;

2. Há influência da pressão sobre o diâmetro interno da tubulação.

3. Os valores de perda de carga determinados experimentalmente e ajustados por análise de regressão, não diferem significativamente, a $5 \%$ de probabilidade, dos valores calculados pela equação de Darcy-Weissbach, com f determinado pela equação de Blasius e diâmetro do tubo corrigido em função da pressão.

4. O coeficiente de redução da perda de carga diminui com o aumento da pressão, para um mesmo número de emissores;

5. Para condições de nível e aclive, o comprimento máximo da linha lateral ocorre para a maior pressão de entrada $(98,1 \mathrm{kPa})$ e, para declive, varia em função da pressão de entrada, do gradiente de declive e do coeficiente de variação de pressão admitido na linha lateral.

\section{REFERÊNCIAS BIBLIOGRÁFICAS}

ABNT-ASSOCIAÇÃO BRASILEIRA DE NORMAS TÉCNICAS - ABNT. Emissores para sistemas de irrigação localizada - avaliação das características operacionais. PNBR 12:02-08-021, São Paulo, 1986, 7p.
ANYOJI, H.; WU, I.P. Statistical approach for drip lateral design. Transactions of the ASAE, St. Joseph, v.30, n.1, p.187192, 1987.

ANDRADE, D.V. de. Avaliação hidráulica de tubos flexíveis de polietileno perfurados a laser, utilizados na irrigação. Piracicaba: ESALQ/USP, 1990. 147p. Dissertação Mestrado

BOTREL, T.A. Hidráulica de microaspersores e linhas laterais para irrigação localizada. Piracicaba: ESALQ/ USP, 1984. 78p. Dissertação Mestrado

CHRISTIANSEN, J.E. Irrigation by sprinkler. Berkeley: California Agricultural Experimental Station, 1942. 124p. (Bulletin 670).

FARIA, M.A. Características hidráulicas do microgotejador IRTEC e da linha lateral de irrigação. Viçosa: UFV, 1981. 72p. Dissertação Mestrado

GILLESPIE, V.A.; PHILLIPS, A.L; WU, I.P. Drip irrigation design equations. Journal of the Irrigation and Drainage Division, New York, v. 100, n. IR4, p. 443-454. 1979.

KELLER, J.; KARMELI, D. Trickle irrigation design. Glendora: Rain Bird Sprinkler Manufacturing Corporation. 1975. 133p.

OLIVEIRA, C.A. da S. Hidráulica de gotejadores e de linhas laterais para irrigação por gotejamento. Viçosa: UFV, 1978. 72p. Dissertação Mestrado

SAMMIS, T.W.; WU, I.P. Crop yield as affected by irrigation. In: INTERNATIONAL DRIP/TRICKLE IRRIGATION CONGRESS, 3., 1985, Fresno. Proceedings... Fresno: ASAE, 1985. p. 22-28.

SCHMIDT, M.V.V. Características hidráulicas do tubogotejador "Queen Gil”. Viçosa: UFV, 1995, 43p. Dissertação Mestrado

SOARES, A. Características hidráulicas de microtubos Cipla e de linhas laterais para irrigação por gotejamento. Viçosa: UFV 1981, 78p. Dissertação Mestrado

SOLOMON, K. Manufacturing variation of trickle emitters. Transactions of the ASAE, St. Joseph, v. 22, n. 5, p.10388. 1979.

TESTEZLAF, R.; CAMPIONI, E.C. Comportamento hidráulico do tubogotejador "Queen Gil". Engenharia Agrícola, Campinas, v. 13, p.29-38, 1993.

USDA - SOIL CONSERVATION SERVICE - Trickle irrigation, Washington D.C. 1979. Cap. 7

VERMEIREN, L.; JOBLING, G. Localized irrigation. Rome: Food and Agriculture Organization of the United Nations. 1980, 203p.

ZAR, J.H. Biostatistical analysis. 2. ed. New Jersy: PrenticeHall, 1984. p.292-305. 